\title{
Stimulus, Expectations, Desires and Response Strategies: The Building Blocks of Nonlinear Interaction Dynamics
}

\author{
DIMITRIOS S. DENDRINOS*
}

School of Architecture and Urban Design, The University of Kansas, Lawrence, KS, 66045-2250, USA

(Received 13 March 2001)

\begin{abstract}
Stimulus and response functions, as the fundamental blocks of nonlinear interaction dynamics, are analyzed and modeled here, under a variety of interaction strategies. The backbone of the suggested model is a simple iterative dynamical formulation: the magnitude of a signal sent at present is a function of a relevant signal sent-as well as of a signal received - in the past; in turn, a signal received at present is a function of a signal received - as well as of a signal sent-in the past; namely,

$$
S_{\mathrm{s}}(n+1)=f_{s}\left\{S_{\mathrm{s}}(n) S_{\mathrm{r}}(n)\right\} \quad \text { and } \quad S_{\mathrm{r}}(n+1)=f_{r}\left\{S_{\mathrm{r}}(n) S_{\mathrm{s}}(n)\right\}
$$

where $S_{\mathrm{s} / \mathrm{r}}$ is the size of a signal sent/received, and $n$ is a point in time.

Expectations and desires by senders and receivers of signals are introduced in the analysis. As a result, a basic classification of individuals emerges, that of "dreamers" and "cynics." Three fundamental response strategies are identified, all plays on a theoretically Newtonian action-reaction system. A pair of individual, as well as community, interaction end-states are the focus of the analysis here; their stability properties, under a tit-for-tat response strategy, are studied and their likelihood to occur is considered.
\end{abstract}

Keywords: Interaction; Individual; Community; Stimulus; Response; Expectation; desire; learning; intent; response strategy; utility; dreamer; cynic; discrete nonlinear dynamics; stability

\section{INTRODUCTION}

An effort is undertaken in this paper to model the dynamics and generic character of interaction among two individuals or agencies. It is argued that there are certain fundamental elements and conditions associated with social interaction dynamics, always encountered in social systems whenever two entities are engaged in some form of stimulus-response exchange. Centrally located is the condition that these dynamics are inherently nonlinear, and that they are the result of various response strategies adopted by social actors. A variation of classical Newtonian type action-reaction, the generic model suggested is broad enough to accommodate a wide range of human interactions. Fields such as micro and macro economics, sociology, political science, social and individual psychology, business and professional practice are among the grounds to utilize this model. Although basic notions from both psychology and economics are employed, the model suggested is neither "pure" psychology or economics, but rather a "composite" social science one.
Much of the foundation for the work here is drawn from prior research by the author, specifically from the paper Dendrinos (1998). There, the basic model of social interaction dynamics was laid out. Here, some significant modifications are made to highlight the aspects of interaction that readers of this journal might find of interest. The action-reaction system, as proposed in 1998, intended to identify the source of positive or negative feedback loops, associated with inherent self-fulfillment or self-defeat aspects of public action. Here, a closer look is taken on the actions by individuals in reference to expectations and desires they possess, as well as to response strategies adopted, in the context of any human interaction. It is argued that qualitatively similar processes underlie private and public actions.

Social psychology, with the study of interaction among individuals prominently figuring at its core, is particularly keen to this framework. For instance, the interaction dynamics characterizing an attraction vs. repelling relationship between two (and possibly more) individuals, vis $a ̀$ vis a response strategy, is quite suitable to the generic model presented below, as are other (professional work,

* E-mail: ddendrinos@ku.edu 
familial, or trade) engagements. Revenge versus forgiveness or compassion, as strategies upon which human interaction is based, can be aptly studied given the generic framework supplied.

A variety of social conditions leading to emotional attachment or detachment of individuals can be approached within the general sketch proposed here. Ranging from dynamics leading to social bonding involving mere friendships, quick marriages or divorces, platonic relationships, lasting mating, to violent crimes of passion, or to other violent forms of social engagement, one could use the suggested framework to analyze social events abundantly found in the fields of social psychology, criminology, sociology, and allied social sciences.

A central aim of the analysis which follows is to demonstrate the inherent aspects of over or under reaction in a two-person/agency interaction. Nonlinear dynamics based coupling almost always contains over or under reaction, often the byproduct of pursuing one (or multiple) stable dynamic equilibrium state(s), or moving away from unstable ones, under an adopted stimulus-response (coupling) strategy.

Excessive quantities of stimulus or response are encountered in a wide variety of forums. Beyond the examples given above, overstimulation (over or under reaction) is also detected in the behavior of stock prices in all stock exchanges, and in almost all aspects of public, economic and social policy. One encounters excesses in responses surrounding the demonstration of human emotions and feelings, such as affection, indifference or repulsion, greed or fear, love or hate. A vast array of dynamic linkages between individuals supply the forum for these excesses to occur. Interactions among nations, leading towards or in avoiding military engagement, supply another context where this framework could be insightful.

Certainly, the central claims of this general model structure are that, first, a fundamental nonlinearity, and second the presence of dynamically stable and unstable multiple end-states characterize human interaction, under any choice of reaction strategy. Chaotic motion is possible. It does not, however, follow that mathematical chaos can be (or is) likely in such inherently nonlinear relationships, a theme which is not elaborated further in these pages.

Due to the systemic nature of the paper and the depth of the subject, the reader is forewarned to expect numerous splinters, the beginning of strings of further and future work. These and other points of bifurcation are not followed here, primarily due to lack of time and space, but merely indicated.

\section{THE STIMULUS (ACTION)-RESPONSE (REACTION) MODEL}

At the outset, the model's elements will be given, followed by the model's conceptual primitives. Then, the model formulation will be supplied together with its dynamics and equilibrium states.

\subsection{The Elements Of the Model and Some Elaboration}

The discussion which follows dwells onto a generic and systemic presentation of the necessary and sufficient elements found in a human interaction, no matter its sociocultural context. With a focus on the central elements, necessary to make the underlying model manageable, certain basic but related themes are outlined. Immediate, and possibly significant, modifications are touched upon as relevant extensions are recommended.

By introducing these additional suggested elements into the model, one could enhance its capacity to deal with far more complicated conditions in human interaction dynamics than those addressed here, where the focus is on individual exchange. Complex interactions are abundant in a social (collective) context. Although the model is based primarily on actions by individuals, it is general enough to accommodate basic components of actions and reactions by governmental agencies as well, at the collective level.

\subsubsection{Individuals, Their Heterogeneity and the Community}

An individual, designated by index $i$ (where $i=1,2, \ldots I$ ), is considered to be part of a set of $I$ different individuals making up a community of interacting entities. Individuals are heterogeneous not only because of the manner in which they interact with other members of the group, but also because of the differing amount of resources and initial endowments they possess to do so. Bound $I$, possibly subscripted by index $i$ and thus considered to be individual specific, is never sharp or constant. Endogenous growth or decline, and in or out migration alter perceived community size in time.

Community composition (the "heterogeneity" factor and the group's perceived size), resource distribution, and interaction are linked in a three-way interface, whereby each affects the other in a cause-effect relationship. At any point in time, there is a resource distribution among members of a community, the exact form of which does depend to an extent on the interactions taking place within the community, as well as the prevailing degree of heterogeneity within the community. Heterogeneity is affected by the resource distribution and the prevailing pattern of interactions within the group. Finally, interaction among the members of the community does depend on the prevailing patterns of differentiation and resource distribution.

Thus, possibly a highly nonlinear interdependency exists among these three elements found in any community. 


\subsubsection{Interaction, Stimulus and Response, and Their Magnitude}

Every individual $i$ in the community interacts in either pair-wise fashion $(i j)$ with all other members of the group, as $j=1,2, \ldots I$, or in a one-to-many context. Interaction takes the form of a stimulus and a response. Each individual member of the community stimulates (or acts upon) members of its group by sending signals. A stimulus by sender $i$ to another entity $j$ is also a response (reaction) by sender $i$ to a previously received signal from $j$, in the context of a continuous exchange.

Thus, there are two basic functions carried out by an individual, that of receiving (and processing) signals, and that of emitting signals to others. Scarce resources are allocated by each individual member to these two basic, but competing, types of activities.

Evidently, an individual (or agency) sends to and receives from each individual (or agency) in the group a variety of signals, at any given point in time. The amount (multiplicity, frequency, strength) and quality of stimuli an individual sends, as well as the amount and quality of stimuli received and processed depend on the available resources the individual possesses and commits to these activities at any point in time. For reasons of mere simplicity, it is assumed here that only a single signal is sent to and is received from another individual (agency). And, only quantity is the element of import, whereas aspects of quality are left to future work. In specific, the varying over time magnitude of a signal sent or received is something that the model proposed here does explicitly address.

\subsubsection{Origin, Destination and the Complement of An Interaction}

Individual $i$ neither sends signals to nor receives signals from all members of the group in uniform strength (size) or frequency. Instead, it discriminates by focusing its primary attention (and resources) on a selected subset of (at least one, nonetheless small in number) individuals in the set $I$. In effect, individual i selectively interacts (competes) with a small subset of the individuals in the group. In sending messages to a signal sink, the individual competes with others for maximum effectiveness of the signal entity $i$ transmits to the desired destination.

At the other end of the linkage, in receiving signals, the individual discriminates among the multiplicity of signals received by concentrating on the one(s) which contribute the most to $i$ 's welfare. In effect, individual $i$ competes with others for attracting a signal from a desired or focal origin, discarding or ignoring signals sent from an indifferent message source.

It is worthy of note that the sending of a signal by one individual to another in a community may or may not have an external effect upon interaction with other members of the community. In the model, which follows, no such external effect is to be considered and consideration of (at times quite important) interaction externalities is left to future work. To make the interaction manageable, a pinpointed (surgical) interaction is assumed, whereby at any point in time individual $i$ wishes to and does send a stimulus to a particular individual $j$ in the community, and wishes to and does receive (and does process) a response from that individual. More precisely, the basis of the model is that the (ij) and ( $j i)$ interactions can be isolated and studied separately from any other $(i k)(k i)$ interaction, where $k=1,2, \ldots I$.

At the same time, individual $i$ interacts with the rest of the members of the group collectively; the individual is linked by lump sum type messages (through sending and receiving such signals) with all other members of the group. The rest of the set members, to be referred to as the "aggregate complement of $j$ " within group of size $I$ (a complement which includes $i$ itself), is to be designated as $c$. Thus, for any $(i j)$ and $(j i)$ interaction there is a corresponding (ic) ( $c i$ ) set of interactions as well.

\subsubsection{Environmental Shocks: Hard, Soft}

Consideration of a focal point $j$ and a complement $c$ for individual $i$ within set $I$ allows for a strict definition of environmental shocks possibly introducing noise or disturbances into an exchange. Environmental shocks either disturb dynamical paths within phase space, or shift dynamical trajectories from one phase space to another in a state variable and parameter space. Shocks are expected to be less frequent than dynamics proper of a system, thus being depicted by slow type changes. They are the source of considerable statistical difficulties in dynamical analysis, when temporal state variable observations are limited.

Two distinct types of shocks can be identified. One category of shocks affecting an interaction $(i j)$ is that which can originate from within the community of individuals and specifically from the subset $c$; one could label these shocks, internal to $I$ and in reference to the $(i j)$ interaction, as "soft". Then, there is the second category of shocks which could come from outside the set $I$ altogether, perturbing the interaction between entities $i$ and $j$, and possibly that between $i$ and $c$; this type of external shock one could characterize as "hard". In the nonlinear dynamic interactions envisioned here, shocks are critical for purposes of testing the robustness surrounding the dynamical stability, at both the individual interacion ( $i j$ ) and the community levels.

\subsubsection{Intent of a signal: To Attract or Repel}

In its emitting signals function, an individual exhibits intent, and thus the sending of messages is the outcome of explicitly chosen response (or emission) strategies. A signal's intent is binary, i.e. it either intends to attract (encourage, reward, give incentives) and thus further engage into, or aims at repelling (discourage, penalize, give disincentives) thus inviting disengagement from an 
interaction. Receiving a signal with either to attract or repel intent causes the recipient pleasure (consent) or displeasure (disagreement). In responding, the recipient of a message sent with intent sends in turn a new signal with an appropriate response measure (this being a choice of strategy issue, to be more fully addressed later).

Thus by reacting, an individual drives an interaction towards desired goals. Steering a socioeconomic system towards or away from specific points in phase space is carried out in such a action-reaction process, a central function of public policy by governmental agencies. Intent is thus central in an interaction process, be that individual or collective interaction.

Ambiguity (or fuzziness) in signals is very much possible but, for the purposes of this paper, it will be assumed away. Instead, on the clarity (sharpness, exactness in sending as well as receiving) of signals is what this model is built. To be consistent, the assumption of clarity in a signal requires that it must be further assumed that there are no errors in perception. An attracting or repelling signal is so perceived by the receiver, and at the same time it is so intended by the sender. Error free interaction is an assumption which can (and must) be relaxed in future work, since differing perceptions of the same signal underscore among other things heterogeneity of individuals within the group. It is shown later how this can be easily accomplished within the framework of the model suggested here.

Intent also implies full knowledge by a member of a group of another member's traits (and more precisely, of the expectations and desires an individual has in an exchange, to be more fully discussed later). Relaxation of the full (and symmetric) knowledge assumption might shed light as to the extent that partial knowledge as well as a distribution of the capacity to learn (intelligence), or inability to do so (ignorance, incompetence), may affect a community's dynamic stability. There are numerous reasons why messages are sent in error (whereby, the intended impact was meant to be different than the actual one). A study into the effects of unintended consequences, due to individual ignorance or limited learning capacity, upon a community's dynamic stability must be informative.

\subsubsection{Space}

Location in space, and the associated socio-spatial friction, is a key component in the decision to discriminate among (in stimulating and responding to) members of a group. A community of individuals is always spread over actual or social space, with individual members envisioned for instance as being distributed at any point in time in a fixed grid pattern, with each (square) grid carrying a different density of individuals from its neighboring ones.

Numerous cellular automaton type algorithms, modeling dynamical spatio-temporal diffusion processes, can be invoked to model such socio-spatial interaction. Strength of a signal emitted towards a particular sink, or received from a particular source, could be (along other factors, some of which are formally addressed in this paper later) an explicit function of distance between the origin and the destination of the message in the (spatial) interaction. Time elapsed between a signal sent and the time it is received could also be a function of distance.

As a result of such spatial considerations, spontaneous spatial configurations can be obtained, in the form of distribution of vacant spatio-temporal spots (and concomitant formation of varying in density spatio-temporal neighborhoods) within the fixed spatial grid pattern. Linked to issues surrounding spatial proximity (access), externalities, and scale effects, all well studied topics in the socio-spatial literature, spatio-temporal aggregate (as well as individual) interaction dynamics underlie the formation of human settlements.

But the emphasis here is not on these aggregate sociospatial interaction dynamics. Instead, by focusing on non spatial individual interaction dynamics, intent and interaction strategies are explicitly explored, elements largely absent from currently available aggregate spatial interaction dynamics models, see for example Bertuglia et al., 1987. Questions regarding the dynamical stability of aggregate spatial interaction patterns can be fruitfully addressed within the framework suggested here, where intent and interaction strategies are fully recognized. Given the audience of this journal, however, the analytical aspects such spatial interaction dynamics are not elaborated and are left to future work.

\subsubsection{Time: Unit Length, Fast and Slow Dynamics}

Time enters this analysis in discrete steps, from some initial point in time $t(0)$, to a time horizon $t(N)=T$, so that any point in time $t(n)$, where $n=0,1, \ldots N$, is found in the period: $t(0)<t(n)<T$. Thus, a system of difference equations is proposed to identify the (nonlinear) iterative interaction dynamics of the system. Although there is nothing in principle to prevent the setup of a continuous time type-modeling framework, consideration of discrete time carries certain advantages. For one, in social sciences data are usually recorded in discrete time steps; and, from a mathematical standpoint, discrete dynamics allow for a far richer menu of dynamical events to be present when dimensionally (specifically twodimensional) small systems are considered.

Following standard analysis, relatively fast dynamics are depicted by the state variables'time sequence, or paths, in phase space. Relatively slow dynamics are depicted by changes in the model's various parameters, i.e. shifts in phase space and/or of trajectories in a particular phase space. Slow dynamics might be the result of environmental shocks, of the hard or soft variety.

Further, the actual size of time unit $n$ depends, of course, on the particular problem under investigation. An economic public policy, associated for example with a change in interest rates implemented by a nation's central 

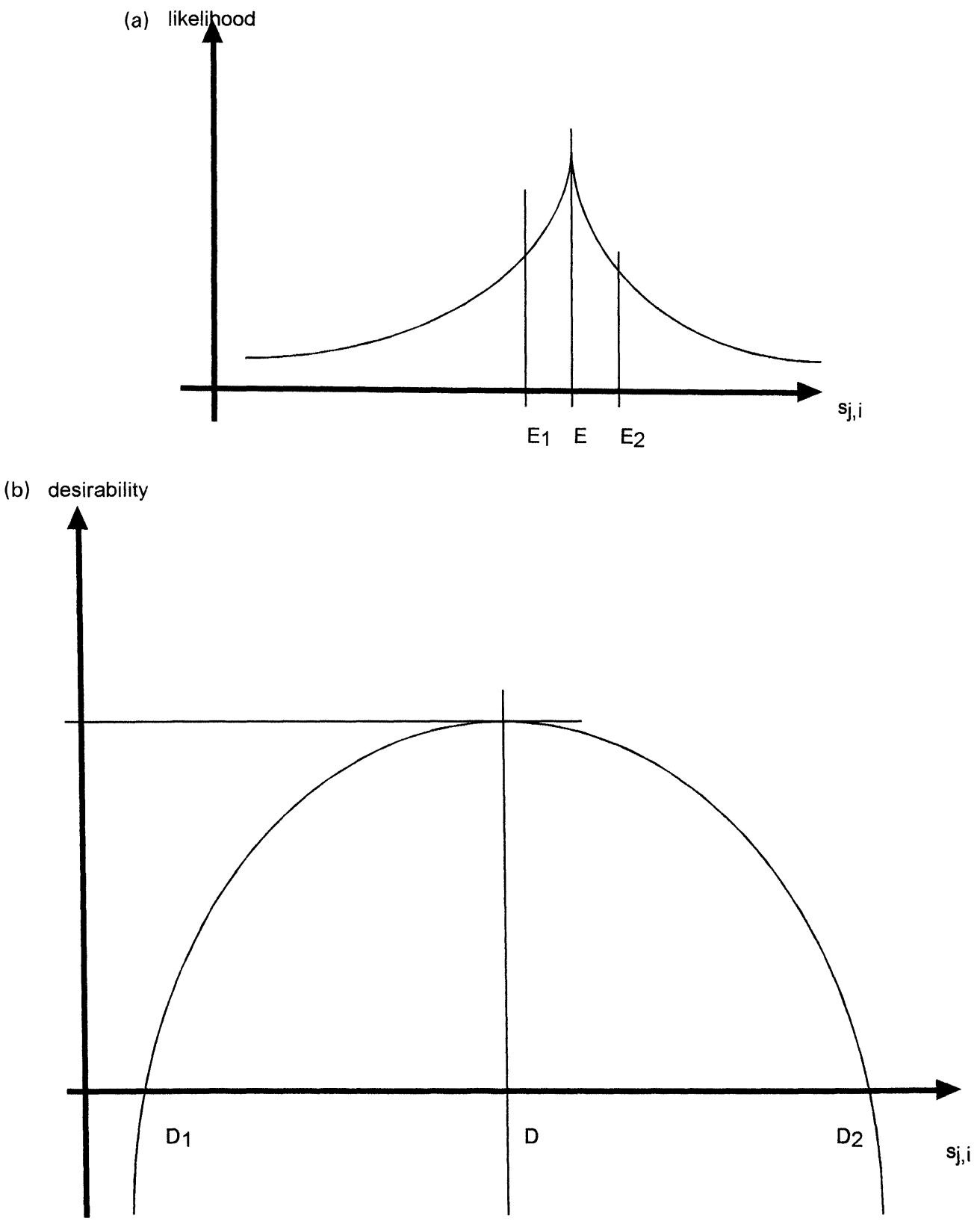

FIGURE 1 (a) The likelihood (expectations) $E$-function of a signal's size. (b) The desirability $D$-function of a signal's size.

bank and intended to stimulate the nation's economy, undoubtedly, will have a different actual time unit length (n) than, for example, a love/hate relationship between two individuals. In the former case, $n$ could last for a month or a quarter; whereas, in the latter, $n$ could depict hours or days. These interactions could possibly differ, as well as to the relevant time horizon $T$.

\subsubsection{Summary on Elements}

At any point in time $t(n)$, individual $i$ carries out two competing in terms of resources actions. First, it receives and processes signals of specific magnitude sent from another individual $j$ and its complement $c$ within community $I$. Second, depending on the effects upon it of the signals (currently, at $t(n)$ ) received, individual $i$ responds during the next point in time, $t(n+1)$, by emitting a set of new signals with intent following a reaction strategy. This action-reaction engagement is depicted by an iterative process, which commences at some initial state.

A starting point for the exchange occurred when an individual commenced stimulating another one within the community, thus setting off a chain reaction in a dynamical interaction context. Initiating an interaction among any pair of individuals within a community, as well as terminating one, is a complex issue of initial and end states (as is always the case in any dynamics context), and 
a topic in its own right. However, the issues involved in uttering the "first" and "last" words in an exchange fall outside the scope of this paper.

This list of elements completes the external (more or less simple, and largely exogenous to the outcome of the model itself) model structure.

\subsection{Model's Conceptual Primitives And Related Discussion}

At a starting point in time $t(0)$, as well as at any subsequent point in time $t(n)$, individual $i$ emits signals towards entities $j$ and $c$, denoted by $s_{i, j}[t(n)]$ and by $s_{i, c}[t(n)]$ correspondingly; at the same point in time $i$ also receives (perceives and processes) signals, denoted by $s_{j, i}[t(n)]$ and by $s_{c, i}[t(n)]$ respectively, originating in entities $j$ and $c$ with a destination towards $i$. The signals' strength (or magnitude) can range from minus to plus infinity; but for reasons of simplicity and without any loss of generality, they will be assumed to vary between zero and plus infinity.

Following the reception of signals and their processing, individual $i$ plans on the basis of a response strategy (to be addressed later) to emit at the next time period a set of two signals of magnitude $s_{i, j}[t(n+1)]$ and $s_{i, c}[t(n+1)]$ correspondingly, to destination entities $j$ and $c$ respectively. The magnitude of these signals, too, varies in the non negative domain of real numbers.

Of interest is the fact that the emission of the response signals carries intent, and follows the adoption of a response strategy by the sender. To address these issues, one must examine the conditions under which the receiver of a signal responds to the effects that the signal received has upon it.

\subsubsection{Expectations, Desires and Their Measures}

Central to the general framework found in Dendrinos (1998) and adopted here is the existence of differing expectations and desires that an individual has in reference to the magnitude of the signals received from another entity. Expectations individual $i$ possesses for signals sent from entity $j$ and the background crowd $c$, at any point in time $t(n)$, are to be designated as $E_{j, i}[t(n)]$ and $E_{c, i}[t(n)]$ respectively. Equivalently, the desired levels individual $i$ has, at time $t[(n)]$, for signals emitted from $j$ and $c$ and directed towards $i$, are denoted by $D_{j, i}[t(n)]$ and $D_{c, i}[t(n)]$ correspondingly.

Below, first a presentation of the concepts surrounding the notion of "expectation" is given, followed by a discussion of those around that of "desire." It is worth noting that fear can also be considered (as being the inverse of desire), so that the analysis which follows can be carried out in terms of fears rather than desires.

\section{EXPECTATIONS}

Expectations are represented by a "signal expecting function," to be referred to as the $E$-function, defined as
$E\left\{s_{j / c, i}[t(n)]\right\}$ over the positive part of the real space, $s_{j / c, i}[t(n)]$. E-functions can be depicted by a likelihood (probability or other, non statistical, for example fuzzy) distribution. In Fig. 1a a particular single peak $E$-function is shown, as an example.

Individual $i$ identifies an "expectations neighborhood" around some expected value of a signal, $E$, to be referred to as the "expectation measure." An expectations neighborhood is delineated by an inner limit $E_{1}$ and an outer limit $E_{2}$, see Fig. 1a, surrounding expectation measure $E$. When an actual signal received (perceived) has a magnitude falling within the expectations neighborhood, then it is thought of as being "more or less expected," with a signal coming at exactly point $E$ (where $s_{j / c, i}[t(n)]=E$, an unlikely "bull's eye."

For the purposes of this model, each of the likelihood functions $E\left\{s_{j / c, i}[t(n)]\right\}$ will be reduced to two expectation measures, $E_{j / c, i}[t(n)]$ These measures could be considered to be the corresponding means of a single hump (either of the type shown in Fig. 1a, or any other, for example, Poisson) probability distribution functions. A simplifying assumption makes analysis quite easier, as in reality an expectations function is not necessarily confined to such a statistical measure. The likelihood function is always positive in the spectrum of (non negative) values of $s_{j / c, i}[t(n)]$ over which it is defined.

Through a process of learning, $E$-functions change in time. How this takes place is central to the model proposed here, and it is addressed in detail in later sections of the paper.

\section{DESIRES}

Equivalently, a "signal's desirability function," the $D$ function, is defined as $D\left\{s_{j / c, i}[t(n)]\right\}$, and it is associated with a specific signal magnitude over the positive part of the real space, $s_{j / c, i}[t(n)]$. $D$-functions can be depicted by any function (among a menu of functions, for example, exponential, logarithmic, logistic, cusp, nonlinear with a single or multiple maxima, etc). The case of a single hump $D$-function is shown in Fig. 1b. Fear bearing behavior can be thought of as the inverse of the $D$-function, so that what follows can also be stated in terms of a Fear-function, something which is left as an exercise to the interested reader.

$D$-functions also change in time, but these changes are more fundamental than those underlying shifts in an $E$ function. Shifts in $D$-functions are related to changes in the traits of individuals within set $I$, factors such as aging, impoverishment or enrichment, or changes in preferences. For reasons of simplicity, it will be assumed that individuals do not change, and that an individual's $D$ function remains constant over time horizon $T$, with an unaltered maximum level at some immobile point $D$.

In contrast to $E$-functions, $D$-functions can obtain both positive and negative values, identifying regimes of "desirability" and "undesirability" in the spectrum of certain signal sizes. Limits $D_{1}$ and $D_{2}$ identify the magnitude of the "attraction region" for signal $s_{j / c, i}[t(n)]$, 
Fig. 1b. Beyond these limits, a signal turns into a repeller in this single hump desirability function case.

At the (single) optimum signal size, associated with a (in this case single) maximum of the $D$-function, target D the first derivative of the $D$-function with respect to signal size $s$ is zero; at that point, further, its second derivative is negative. These are the first and second order conditions for (local or global) optimum. A positive (negative) exponential form of the $D$-function, a higher (lower) level of signal $s$ always implies a more (less) desirable state. Under this functional form, the first and second order conditions fail: plus infinity (zero) is the optimum level for $s$.

To give two examples of a target, a personal relationship and a macro economic indicator policy are evoked, at the individual and collective levels. A target can identify, in the case of an individual $i$, the desired magnitude of affection returned towards it from another individual $j$ in the community. At the level of a public body, a target could identify a desired level of interest (or unemployment, inflation, or economic growth) rate an agency like the Federal Reserve Board could pursue.

\subsubsection{Knowledge, Ignorance, Learning}

The sender of a message may know exactly, approximately, or not at all the form of a receiver's two trait functions (expectations, $E$-function, and desirability, $D$ function), and their various measures, $(E, D)$. The degree to which these functions, characterizing a receiver, are known to the sender of a signal implies a level of knowledge (or competence) of the receiver by the sender. Knowledge is the outcome of a learning process, of the receiver by the sender. Further, knowledge is a characteristic, on the average and at any point in time, present in the community among its members. It is to be explicitly addressed here. Competence on the other hand is a function of an individual's level of intelligence (and thus a part of the initial endowments). Treatment of competence and its changing levels in time is left to future research.

Under a symmetry (or reciprocity) assumption, one can consider that in an exchange [(ij) and (ji)] the knowledge possessed by any of the two members about the other is identical. That is, if individual $i$ knows with some probability the approximate measures $(E, D$ and their distribution) for entity $j$, then in turn entity $j$ knows with the same probability the corresponding measures (and distributions) for individual $i$. How is the symmetry assumption introduced in the model, and how it is possible for the impact of its relaxation to be gaged, are topics discussed at length later in the paper.

Ranging from full knowledge (error free or high skill level) to total ignorance (erroneous, complete incompetence or low skill level) one is confronted by a spectrum over which individuals might be distributed. Density of knowledge (or ignorance) might be critical in the study of the community's dynamical stability. In turn, the extent of knowledge (error or ignorance) available might be associated with the degree to which the community comprises of similar or diverse individuals. So, another link is found between diversity of a community and its eventual dynamical stability (or instability).

Most importantly, however, knowledge of these functions and measures characterizing entity $i$ by entities $j$ and $c$, and actions by $j$ and $c$ in sending signals (against the background of these functions and measures) towards $i$, imply that the sender carries intent in transmitting signals. Full knowledge of these functions characterizing $i$, by $j$ and $c$ would imply that there are no unintended consequences. Whereas, partial knowledge of these functions imply that possible misinterpretations of signals can occur. The manner in which intent is incorporated into interactions will be explore later in this paper (in the model formulation section).

\subsubsection{Independence Of E/D-functions}

Going one step further, the functions and measures characterizing any entity of an interaction within the community are assumed to be independent of the other members' functions and corresponding measures. Again, diversity (as opposed to homogeneity) within a community might be a determinant of such interdependencies: the more diverse the individuals in a community, the weaker the force compelling all to converge towards similar functions and measures; the more homogeneous the individuals of the group, the stronger the force to assimilate under a single $E / D$-function and associated measures.

Were one to introduce such interdependencies (or externalities), then the dynamics would become unnecessarily complicated and even more nonlinear. They would enhance the possibilities for the model to exhibit highly unstable dynamic patterns. But they would not negate the key tenants of this study and its conclusions, except to reinforce the ones to be demonstrated.

\subsubsection{Acceptance, Rejection in Interaction}

A signal's interpretation by entity $i$ is obviously linked to the desired magnitude of the signal coming from a particular source $j$. Were individual $i$ to desire an appreciably different magnitude signal from $j$ than the one received (smaller or bigger in size than the target $\mathrm{D}$, in any case not in the region $D_{1} D_{2}$ of Fig. 1b), it will be constructed by $i$ as a repelling sign. In effect, it is a message for $i$ to change interaction strategy.

If the level of the signal received still produces a positive level of desirability, but still is not very close to the optimum (target) signal size, then individual $i$ might not be motivated to change its interaction strategy entirely. But, individual $i$ will certainly be mildly disappointed, and thus appropriately respond. It can be asserted that, in both cases, individual $i$ has felt a level of rejection. On the other hand, were the received signal's actual magnitude close 
enough to (in the very near neighborhood of) the desired level $D$, then $i$ must interpret the signal as enticing further engagement in an on going interaction. Individual $i$ must feel a great deal of acceptance out of this exchange.

A slightly different look at the neighborhood $D_{1} D_{2}$ might reveal the presence of a "security" enclave of signal magnitude for entity $i$ : if an actual signal is received and falls within this enclave, then it may make $i$ feel secure about $j / c$; whereas, a signal carrying a magnitude outside this zone might induce within $i$ feelings of insecurity and possibly fear or even anxiety, depending on the distance of the actual signal's magnitude from the comfort zone.

\subsubsection{Success, Failure in Interaction}

Comparing the level of an actually received signal to that of its expected value gives the receiver information about the degree to which an individual's calibrating mechanism of the interaction model is working properly. Failure is registered by the receiver when the difference between the two is relatively large in absolute value, with a negative balance implying a sense for $i$ of being "overwhelmed" by $j / c$, and with a positive balance signifying a sense of being "underwhelmed". Whereas, if the difference falls within acceptable limits, then this is a sign for success for $i$ in sizing up $j / c$.

By measuring, in absolute value, the deviation between the magnitude of the actual signal received $s_{j, i}[t(n)]$ and the expected level of the signal, $E_{j, i}[t(n)]$, one picks up the level of failure (or lack of success), indicated as $p_{j, i}[t(n)]$, of individual $i$ to accurately predict $j$ 's actions. Similarly, with regards to the background, the difference in absolute value between $s_{c, i}[t(n)]$ and $E_{c, i}[t(n)]$, to be indicated as $p_{c, i}[t(n)]$, is indicative of the degree to which individual $i$ failed to gage the action of the background crowd.

Further, by an equivalent measure, one can identify the rejection (or non acceptance) level experienced by entity $i$, simply by looking at the difference (in absolute value) between $s_{j, i}[t(n)]$ and $D_{j, i}[t(n)]$, as well as between $s_{c, i}[t(n)]$ and $D_{c . i}[t(n)]$, to be designated as $d_{j, i}[t(n)]$ and $d_{c, i}[t(n)]$ correspondingly.

\subsubsection{Disutility, Utility, Response Strategies: Purpose in Interaction}

Both indices $(p, d)$ measure disappointment levels experienced by individual $i$ as a result of signals flowing from $j$ (and/or $c$ ) towards $i$. These one-way (from $j / c$ towards $i$ ) sources of displeasure for $i$ are different in nature. Combined and appropriately weighed, they identify the disutility experienced by individual $i$ at time $t(n)$ by being engaged within the community by $j$ and $c$, to be designated as ${ }^{-} \mathrm{U}_{i, j}$ and ${ }^{-} \mathrm{U}_{i, c}$, correspondingly. "Utility" and "disutility" are used here approximately as they are employed in a neoclassical micro economics context. Specifically,

$$
\begin{aligned}
& { }^{-} U_{i, j}[t(n)]=\alpha_{1} p_{j, i}[t(n)]+\beta_{1} d_{j, i}[t(n)] \\
& { }^{-} U_{i, c}[t(n)]=\alpha_{2} p_{c, i}[t(n)]+\beta_{2} d_{c, i}[t(n)] .
\end{aligned}
$$

where coefficients $\alpha, \beta$ are non negative weights, $\alpha$ being converters of disappointment (due to inability to properly gage a signal) into disutility, and $\beta$ being converters of unhappiness (due to rejection) into disutility. One can equivalently define quantities ${ }^{-} U_{j, i}$ and ${ }^{-} U_{c, i}$ as well.

That entity $j / c$ is currently, i.e. at $t(n)$, expected by $i$ to experience a (low or high) level of disutility ${ }^{-} U_{j / c, i}[t(n+1)]$ at the next time period due to $i$ 's upcoming response, causes current pleasure (or utility) to $i$, the level of which is to be designated as ${ }^{+} U_{i, j / c}[t(n)]$. No matter the expected (positive or negative) impact of a signal from a sender towards the receiver, the sender always emits a signal to any destination that causes at present some pleasure to the sender proper. Thus, the purpose of a signal is twofold, to inject some degree of future pleasure (more accurately, displeasure) to the receiver, and at the present time period to satisfy the sender in doing so. In effect,

$$
\begin{aligned}
& { }^{+} U_{i, j}[t(n)]=\gamma_{1}^{-} U_{j, i}[t(n+1)] \\
& { }^{+} U_{i, c}[t(n)]=\gamma_{2}^{-} U_{c, i}[t(n+1)]
\end{aligned}
$$

where translator coefficients $\gamma$ are non negative quantities; they act as converters of utility units for entity $i$ of utility units of entity $j$ or $c$.

In responding with intent, entity $i$ is assumed to possess the requisite knowledge of $j / c$ 's current expectation and desire measures. Further, in responding with intent, entity $i$ adopts an appropriate response strategy towards $j / c$. Response strategies link the current disutility levels experienced by $i$ from receiving a signal from entities $j / c\left({ }^{-} U_{i, j / c}[t(n)]\right)$ to the currently expected by $i$ future disutility of the receiver $j / c$ due to the upcoming response $\left.\left({ }^{-} U_{j / c, i}[t(n+1)]\right)\right)$.

There are numerous response strategies an individual could choose from in reacting, a few possible ones listed below:

Under a tit-for-tat response strategy, individual $i$ is motivated to respond by inflicting upon entities $j / c$ an amount of displeasure approximately equal to the one received; this is the case when the following conditions hold:

$$
\begin{aligned}
{ }^{-} U_{j, i}[t(n+1)] & ={ }^{-} U_{i, j}[t(n)] \\
{ }^{-} U_{c, i}[t(n+1)] & ={ }^{-} U_{i, c}[t(n)]
\end{aligned}
$$

so that

$$
\begin{aligned}
{ }^{+} U_{i, j}[t(n+1)] / \gamma_{1} & ={ }^{-} U_{j, i}[t(n)] \\
{ }^{+} U_{i, c}[t(n+1)] / \gamma_{2} & ={ }^{-} U_{c, i}[t(n)] .
\end{aligned}
$$

Under gradual response strategy individual $i$ would be motivated to respond by causing $j / c$ a multiple of the amount of displeasure caused $i$; this occurs when

$$
\begin{aligned}
& { }^{+} U_{i, j}[t(n+1)] / \gamma_{1}=w^{-} U_{j, i}[t(n)] \\
& { }^{+} U_{i, c}[t(n+1)] / \gamma_{2}=z^{-} U_{c, i}[t(n)] ;
\end{aligned}
$$


when coefficients $(w, z)>1$ escalation is implied, while if $(w, z)<1$ a form of diffusion is meant to characterize the exchange; the closer to zero the value of these two parameters, the more indifferent the mode of interaction is; Obviously, when $(w, z)=1$ then one has the tit-for-tat response strategy;

Under a blind response strategy, individual $i$ would tend to act towards $j / c$ regardless (independently) of the displeasure level caused $i$ by their signals, i.e. when $w, z=$ 0 it is implied that $i$, in responding to $j / c$, is indifferent as to the impact that $j / c$ 's signals had upon $i$.

All three types of response strategies are plays on classical Newtonian action-reaction systems, the exact physics of which is a subject of possible future work. Moreover, the conversion of a human (two-actor, or manyactor) interaction strategy from classical Newtonian to relativistic or even quantum type is also something of considerable interest, but outside the scope of this paper. Nonetheless, these realizations provide a valuable link between social and physical systems, analyzed under the rubric of nonlinear dynamics, a potentially key concern for the readers of this journal.

Tit-for-tat, gradual (escalating, diffusing) and blind response strategies are possible archetypical strategies that seem to cover in essence the spectrum of all response strategies available to an individual in the context of an interaction along the lines of traditional Newtonian analysis. However, whether this is indeed so or not, is a question, which falls outside the scope proper of this paper, as it focuses on results of interaction rather than on the strategies for interaction. For example, there are possibly interesting combinations of values in $\gamma_{1}$ and $\gamma_{2}$ that, in conjunction with actual $\mathrm{p}$ and $\mathrm{d}$ levels would constitute different variations of the above three types of response strategies. All this is left to future work.

\subsubsection{Habits}

Every individual (and agency, in the case of a public entity) is subject to a large degree to habitual behavior, whereby what one does at present is connected to what one has done in the past. Being the source of momentum in human action, habits contribute directly to pleasure and utility (or disutility).

Stated in terms of disutility, a component to be designated as ${ }^{-} U_{l, t}$, one can reasonably assume that displeasure due to habit is given by the expression:

$$
\begin{aligned}
{ }^{-} U_{i, t}[t(n+1)]= & \delta \sum_{j / c} \mid s_{i, j / c}[t(n+1)] \\
& -s_{i, j / c}[t(n)] \mid
\end{aligned}
$$

that is, it is a function of the absolute value of the difference between the signals sent at $t(n)$ and that at $t(n+1)$, the smaller the difference the less the disutility (thus the greater the utility) due to habit. Habitual behavior could be a source of impediment to dynamical adjustments, thus acting as a force counter to violent oscillations in signal magnitudes over time. Habits in effect act as brakes or as a dumping force to cycles.

Habitual behavior is one among the keys which render the interaction dynamic in time, as it links present behavior to past (or, equivalently, future action to present one). Since action and reaction by entities $i, j$ are shown to be simultaneous in the model which follows, addiction together with the process by which expectations are formed are the sole links in inter temporal action within the framework of an exchange. Of course this need not be so in a more complex model formulation.

\subsubsection{Motivation to Interact}

Given the (three) different elements of pleasure (one) and displeasure (two) enjoyed at $t(n)$ by an individual in any interaction, namely ${ }^{-} U_{i, j / c}[t(n)],{ }^{+} U_{i, j / c}[t(n)]$, and ${ }^{-} U_{i, t}[t(n)]$, one can identify the total utility level $U_{i}$, enjoyed by an entity $i$ at any time period; it consists of the sum of specific displeasure (disutility) and pleasure levels; vis $a$ vis the two sources of discomfort and satisfaction found in any interaction ( $i j)$, and addiction to behavior by actor $i$ :

$$
\begin{aligned}
U_{i}[t(n)]= & \sum_{j / c}\left\{{ }^{+} U_{i, j / c}[t(n)]-{ }^{-} U_{i, j / c}[t(n)]\right\} \\
& -{ }^{-} U_{i, t}[t(n) .
\end{aligned}
$$

Deviations from expectation and desire levels, experienced by an individual as a result of a signal received, combined with one's ability to either reward or penalize the sender by a subsequent signal to be emitted, and addiction to a particular interaction type determine the overall utility for acting in time. Expected changes in this overall utility level reveal the incentive (or more precisely, the level of disincentive) to keep interacting. Motivation, or inducement, to act (and react) so that one attains over time a particular utility level, gives the underlying fundamental purpose in human interaction.

\subsubsection{Dreamers and Cynics: The Pure and Hybrid Variety}

Of particular interest is the difference between the expected (through the value of the $E$-function) and desirable (through the value of the $D$-function) levels an individual $i$ carries in reference to a signal sent from another individual $j$. To be denoted as the ED-gap, a positive or negative quantity, this difference is defined at any signal size. As it is assumed that these two functions can be substituted by their corresponding unique levels ( $E$ and $D$ respectively) for any individual $i$ in reference to another entity $j$, one may profitably examine the meaning of the $E D$-gap.

If the $E D$-gap is relatively small in absolute value, then one may characterize individual $i$ as falling under the "dreamer" category in reference to entity $j$. In this case, 
the individual is seen as the carrier of "great expectations," since what one desires and expects are relatively close. Whereas, if the $E D$-gap is relatively large in absolute value, then the individual is a "cynic," as what one likes and what one expects are far apart.

Employing various combinations of holding expectations and desires, one could describe individuals with expectations but no desires; with desires but no expectations; and finally, without either expectations or desires in an interaction. All these variations are left to future analysis.

Were one to be dreamer or a cynic in reference to all members of a community, and not just $j$, then one may be characterized as a "pure" dreamer or cynic. One could be though as a "hybrid" dreamer or cynic if $i$ is a dreamer or cynic in reference to $j$ but cynic or dreamer in reference to $c$.

\subsubsection{Dynamical Stability and Types of Individuals}

A legitimate set of research questions arise as a consequence of the above assertions: is it the case that most communities studied during their mostly stable states, contain exclusively or in great abundance individuals who are (or must be) of the small $E D$-gap (pure great expectations) variety?

Since not all individuals ought to be expected to fall under either category, one can ask: is there a threshold (share) of individuals that must fall under either of these two types for the community to be stable?

Most importantly, if one assumes that an individual could be a pure cynic at some point(s) in time, but switch to having pure great expectations at a subsequent time period, is there a temporal time frame within which switching from one to the other type by even a few individuals might destabilize community dynamics?

Is it safe to assume that individually stable interactions take place among either pure dreamer/cynic pairs? And to what extent, are hybrid types likely to bond into dynamically stable interactions?

Undoubtedly, sound models of individual interaction, as well as community dynamics, ought to be capable of accommodating such diversity and switching. Along the many research hypotheses that such issues bring about, the question also rises: do stable socioeconomic systems require that policy makers be predominantly cynics? Or, the converse, why does it seem to be the case that cynicism is a viable (stable) evolutionary strategy?

The distinction between "great expectations" and "cynicism" in individual behavior, at any point in time, is drawn from a simple look at the sign and size of the $E D$ gap, irrespective of the magnitude of the actual signal received. Now, the actual magnitude of the signal received will enter the analysis, to further classify individual behavior by linking actions and reactions (the "before" and "after" the action responses) to individual type. It is recalled that entity $i$ responds, during the next point in time $t(n+1)$, to the presently received at $t(n)$ stimuli from either $j / c$. The response is always related to the differentials between the currently expected and desired levels of stimuli and their actual magnitudes, as it will be formally stated in the next section.

\subsubsection{Realism, Utopia and the Dreamer}

Were a dreamer with great expectations to receive an actual signal much different that the one expected (and desired), then clearly one must classify the receiver as carrying "utopian" or unrealistic expectations. The recipient of such a signal must obviously feel disappointment or even despair, depending on the size of $p$, and at the same time be greatly surprised. Vengeance, by adopting the nastiest possible response strategy, is likely to be the reaction by a disappointed dreamer.

Examples of societies, and historical periods, where utopian thoughts initially prevailed among a few carriers of great expectations, albeit for a brief time period, only to be followed by periods of disillusionment, are not entirely rare. In early revolutionary periods, during different eras in human history, certain social systems were dominated by a few fervent dreamers, fanatics, revolutionary extremists and the like, carriers of strong utopian ideals, which only failed in short order. Under these circumstances one could describe these social systems as having been severely unstable.

On the other hand, were the same type individual to receive (perceive) an actual signal close enough to the magnitude expected (and desired), then the individual must be thought of as having had "realistic," albeit great, expectations. The individual must feel exuberance or at least satisfaction depending on the magnitude of $p / d$. The most rewarding of all response functions must be the reactions strategy of choice under these conditions.

\subsubsection{Pragmatism, Surprise and the Cynic}

Different is the picture when a cynic is involved. Were the cynic to receive a signal very different in magnitude than the one desired but close enough to the one expected, then the cynic can be considered to be also a "pragmatist". Were the opposite to be the case (the signal coming close to the one desired, but far from the one expected) then the cynic is clearly "out of touch" with reality. However, in either cases, the cynic does not necessarily feel disappointed, as some hedging has taken place; it certainly does not experience surprise.

But neither does the cynic ever feel elated, as any small $p / d$ is countered by a sizable $d / p$. If the signal received by a cynic is far from both desired and expected levels (an event which is unlikely as the spread due to the $E D$-gap of a cynic covers a lot of ground in the signal's spectrum), then a rare event is recorded: one has encountered a "disappointed and surprised cynic!", the degree of disappointment and surprise directly linked to the magnitudes of $p$ and $d$. 
A response at $t(n+1)$, given a signal received at $t(n)$ from $j / c$, would motivate a cynic to act differently, depending on the relative location of $s$ in reference to $D$ and $E$.

There are three possibilities, two of them having the signal falling beyond both measures, and one accounting for the signal falling between them. The cynic will react by adjusting the signal size emitted differently, depending on each of the three cases. In any case, one should not expect a cynic to choose extreme positions in terms of a response strategy, as opposed to the dreamer's case.

\subsubsection{Rational Expectations: Pessimists, Optimists}

Generally, and regardless of the type an individual is, one can talk about rational expectations only after an exchange has taken place. Only then, can one accurately compare the magnitude of an expected signal before the reception with the magnitude of the signal after its reception. If the difference is approximately zero, then one could talk about "rational" expectations, whereas if the difference is considerably greater than zero, in absolute value, then one could characterize individual $i$ 's expectations as "non rational." This definition is at variance with the definition of rational expectations in the field of economics, see for example Lucas (1984).

Moreover, if the magnitude of the actual signal received is greater than the expected one and also greater than the one desired, then individual $i$ proved to be a "pessimist", since entity $i$ was carrying an "underestimate" (the case of overshooting by entity $j$ towards $i$ ). Whereas, if the magnitude of the actual signal is less than its expected size and also less than the desired one, then individual $i$ proved to be an "optimist", as $i$ was the carrier of an "overestimate" (the case of undershooting by entity $j$ towards $i$ ).

Pessimism, as well as optimism, characterizes conditions where the actual signal clearly falls below or over $D / E$ levels. Mixed feelings of pessimism or optimism appear when intermediate cases occur, whereby the actual signal's magnitude comes between expected and desired levels. These cases imply mixed over or undershooting conditions by $j$ towards entity $i$.

Response strategies adopted by optimists, at any particular point in time must be different than those that could be adopted by pessimists. In both cases, entity $i$ 's response to both $j$ and $c$ at time $n+1$ is partially pegged to the magnitudes of $p$ and $d$, and in specific to the position of $s$ relative to the location of measures $E$ and $D$ in the positive real space. These differentials form part of the basis for specifying the kinematic conditions of the model.

\subsubsection{Potentials}

In the context of any interaction, an individual would engage in an exchange and act so as to maximize overall utility (or, equivalently, minimize overall disutility) level, at any point in time or over a pre-specified time period.
That is, depending on the degree of myopia or foresight, either by a choice of a signal sequence in time the individual could maximize a stream of utilities, or, more likely, the individual would maximize current utility level by a choice of current signals. Whatever the guiding principle, one could profitably assume that a utility maximization process (subject to the resource constraints already mentioned) would act as an operating "potential," giving rise to the specific kinematic conditions of the model. This would imply that:

$$
{ }_{\max } U_{i}[t(n)]=\text { Const }_{i},
$$

where, $\max U_{i}[t(n)]$ is the maximum possible level that could be obtained at $t(n)$, and Const ${ }_{i}=H_{i}(T)$ could be some utility level $H_{i}(T)$ associated with individual $i$, prespecified over a time horizon $T$.

In case maximum utility levels vary in time, it is immediately obvious that temporal utility differentials identity various levels in the motivation to act in time; i.e. the first derivative of $U_{i}[t(n)]$ with respect to $n$ is the willingness to act at $t(n)$.

Along classical Newtonian mechanics, implications from condition (10) might indicate that there is some "energy" condition present in any interaction. Whether an individual is a cynic or a dreamer would determine to a great extent the level of energy present in the interaction, an a priori "passivity or activity" level, such that the actions taken by any individual at any point in time may leave the level of such energy constant. Furthermore, one could argue that there are different initial levels of energy for the cynic (Const $\left.{ }_{\text {cynic }}\right) i$ and dreamer $\left(\right.$ Const $\left._{\text {dreamer }}\right) i$ types; in turn, one could allow individuals to switch energy levels (and thus roles), as they evolve within a community interaction dynamics framework. They could be, among other factors, attributed to the $E D$-gap size. All that is left, however, to future research, a quite promising avenue of work since it may reveal ties between social behavior and natural systems dynamics.

\subsubsection{Summary of Model's Conceptual Primitives}

Split between dreamers and cynics, realists and utopians, optimists and pessimists, individuals act and react on the basis of expectations and desires. By selecting a response strategy, the sender of signals penalizes or rewards the intended receiver(s). In interacting, an individual undergoes three different types of experiences, one of them involving a positive utility and two of these elements identifying a negative utility (disutility) measure.

On the positive utility (satisfaction) side, rational behavior leads a sender to experience pleasure due to the sending of a response signal with an expected (and assumed always to be to the liking of the sender) impact on a receiver. On the negative utility (disutility) side, first, due to addiction in behavior, the difference in the signal sent at present from a prior signal creates displeasure as it contains a brake with habit and tradition; and second, any 
signal received causes the receiver a varying level of pain, since it always deviates from expected and desired measures (unless it is an unlikely bull's eye on both counts).

Responses by senders of signals to receivers are pegged to the specific response strategies available to the sender and adopted in a particular interaction. Three types of response strategies seem to constitute the basis for formulating a menu of alternative response avenues. They range from a tit-for-tat, to gradual (escalating or diffusing) response, to a blind one (total indifference).

Probable potentials in place guide the motivation to interact for dreamers and cynics. Change in the level of these (energy) constants may cause switching from one mode (dreamer) to another (cynic). Individual and community interaction dynamics are linked to the distribution of individuals between these two categories of behavior.

\subsection{The Model's Formulation}

In this section an effort is made to specify the model's form and the underlying equations of motion in the system. Resource constraints with which an individual is limited in receiving, processing and sending signals are not formally elaborated in this simple case.

Setting the model's structure, a few structural issues need to be addressed at the outset, namely the presumed time delays incorporated into the dynamics of the interaction, and the assumptions regarding learning, knowledge base and their symmetry conditions in the two-way exchange. These issues are addressed in turn next.

\subsubsection{Time Delays}

Action and reaction by both members of an exchange are assumed here to be simultaneous, i.e. they do not involve directly a time delay in them. This is indeed a strong assumption particularly when spatial (or social) distance is considered. Nothing, however, requires this to be necessarily the case, except to render the model more complex. Instead, the simple model suggested here relies exclusively on addiction and formation of expectations for its dynamics.

Thus, the first consideration having to do with time delays surrounds in effect the element of habit or addiction. Time delays are also linked to memory and learning through the process of forming expectations, the longer the time delays built into the model the longer the memory and more the amount of learning accomplished. By incorporating either time delays (i.e. memory) into current action, that is by linking the next time period's signal magnitude to the current as well as past signal sizes, one renders history relevant and at the same time weighs the presence of habitual behavior.

Extent of time delay (indicative of how much from the past a current action draws) determines the degree to which history matters to an actor. One could also make the extent of time on which an action is based a function of the type of individual one examines: a dreamer, for instance, could draw from a very long history; while a cynic might confine the extent of temporal consideration to a very short term.

A community obviously consists of members with different (short and/or long term) memories, learning capacities and habits. To what extent average memory length, learning capacity and habitual behavior must affect the dynamic stability of a community (along side individual interaction dynamics) is another research question of great interest left to future undertakings.

\subsubsection{Learning, Knowledge and Symmetry in Interaction}

By learning, this model recognizes the fact that, first, an individual's expectations adjust in time; and second, that through knowledge, an individual's measures of expectations and desires can be better gauged by another entity in the community. Full knowledge implies that an entity has an exact gage of another individual's measures (and $E / D$-functions). Whereas, under partial and ever improving knowledge (through effective learning), an individual's gage of another individual's measures of $E / D$ are approximate. Under symmetry conditions, an individual knows as much about another individual as the latter knows about the former. Whereas, under asymmetric knowledge, an individual's knowledge of another individual's measures is individual specific in its approximation.

A key point of this paper is this: the interaction itself governs a truly evolving system of engagement among the agents or agencies involved.

In the field of economic policy this implies that the perceptions policy makers have of the system subjected to policy making (and vice versa) is not rigid, but an evolving one. Rather than consider the knowledge base of a system fixed from some initial time period, when intervention is initiated, and lasting up till the end of a time horizon while interaction lasts, knowledge level of a system is considered to be in a state of flux. Further, the policy maker's expectations of how the system is likely to behave also change in time.

In the field of social psychology, an individual's response to another individual over time is not pegged to an initial (set at some original time period) knowledge or ignorance level an individual has of another individual's expectations and desires functions. To the contrary, it depends on the responses received by the individual in question to its initial and on going overtures. Put differently, the time sequence of stimuli and responses changes the knowledge base of the action itself, through some learning.

This is a major point distinguishing this analysis from previous ones, (see for example that by Rinaldi and Gragnani, 1998, for an interesting presentation). Rinaldi and Gragnani put forward a model of simulating attraction 
among individuals in pair wise love interaction dynamics. But, an at least questionable and possibly flawed notion is advanced; namely, that all individuals in a community can be ranked by some index of "attraction," which is assumed to be fixed over time, and above all independent of the individual to whom a person might be attractive or not. Moreover, this ranking is commonly accepted within the community! System dynamics, and individual as well as community "equilibrium" conditions are critically associated to the existence of such a robust ranking.

It is the centerpiece of this paper that instead of a strict attractivity ranking, and among other numerous conditions, the likelihood and robustness of a community's stability must critically depend on the relative current (and ever changing) abundance of individuals categorized as "dreamers" or "cynics" within the community. And community stability must also depend on the distributions of the ED-gap, and the $p$ as well as $d$ sizes, among the members of the community, at any point in time $t(n)$. And above all, it must depend on the choice of response strategies.

\subsubsection{Kinetics Under a tit-for-tat Response Strategy}

As alluded to earlier, one would expect inter-temporal utility functions (preferences), a variety of relevant constraints, including initial endowments etc. to determine the functional form of the kinetics. Utility functions would propel individuals to pursue interaction strategies, emit messages and pursue learning, which maximize total utility in an exchange. This is attained by choosing signals that maximize the pleasant and satisfactory experiences, and by minimizing disutility due to unpleasant and unsatisfactory signals received through learning and an improved knowledge base subject to resource constraints. Since resource constraints are not explicitly incorporated into the interaction dynamics modeled here, the kinetics are solely based on an unconstrained utility maximization process.

No matter how objectionable one might consider the simplifying assumptions behind the specifications that follow, they do not even compare with the more or less arbitrary arguments found in other papers (in the various fields of Psychology which attempt to deal with the topic of human interaction). For example, in the paper by Rinaldi and Gragnani (1998), psychological processes associated with "return" "instinct" and "oblivion" are introduced into the governing set of differential equations of motion. The three variables are adopted after the work by Bowlby $(1969,1973,1980)$ and because they were espoused by a number of other psychologists, according to Rinaldi and Gragnani. Use of such ambiguously defined, and largely arbitrary, variables is not needed. One does not have to incorporate these notions into the system's kinematic conditions to obtain the main messages sought. Parsimony is pursued in this model, an attribute not always appropriately valued in social sciences (including such fields as economics, psychology, political sciences, or sociology).

In planning at $t(n)$ and reacting at $t(n+1)$ individual $i$ considers the adverse effects upon it of actions taken by $j / c$ at $t(n)$ directed towards $i,{ }^{-} U_{j / c, i}[t(n)]$. Also at $t(n), i$ enjoys the satisfaction of the intended reaction, ${ }^{+} U_{i, j / c}[-$ $t(n)]$, as well as the satisfaction from addiction to a certain signal size emitted (i.e. the dissatisfaction from their variance in magnitude), ${ }^{-} U_{i, t}[t(n)]$. The combined effects of both types of time

$$
\begin{aligned}
& \sum_{j, c}\left\{{ }^{+} U_{i, j / c}[t(n)]\right\}-{ }^{-} U_{i, t}[t(n)] \\
& -\sum_{j, c}\left\{{ }^{-} U_{j / c, i}[t(n)]\right\} \\
& =H_{i}(T)
\end{aligned}
$$

for individual $i$. Correspondingly, the utility and disutility functions, as well as the action and reaction for individual $j$ towards $i$ are obtained by interchanging $i$ with $j$ in the above condition. Whereas, that from the component $c$ towards $i$ or $j$, as well as from $i$ or $j$ towards $c$ are obtained by suitable substitutions.

This interaction model is the broadest possible (general) formulation of a standard (spatial as well as non spatial) interaction model, involving temporal flow from an origin $i$ to a destination $j$. Kinetics are the direct result of addictive behavior, and indirect result of response strategies and learning.

Next attention is focused on the expectation measures, $E$, and desirability measure (target), $D$. It is reasonable to assume that central in their definition must be a (limited) history of actions, or more exactly, a (cumulative) history of the stimuli received by an individual from a source. Thus, expectations must be some type of a statistical measure of the past $s$ 's, extended over a pre-specified time period in which the volatility of the signal's size is discounted. Under the simplest possible statistical mean form, it can be assumed that:

$$
\begin{aligned}
E_{j / c, i}[t(n)] & =1 / M \sum_{m} s_{j / c, i}[t(m)], m(<n) \\
& =0,1, \ldots M
\end{aligned}
$$

where, expectation related memory index $m$ obtains some maximum value $M \ll N$. The precise definition of $E$, however, is immaterial for the purposes of this work. Given that in this formulation expectations are described by a dynamical path, since expectations are moving in phase space, one confronts the possibility that someone who started as a dreamer at some initial point in time, is gradually (or abruptly, depending of the form of the moving expectations path) transformed into a cynic through the evolution of the community interactions. As already mentioned, measures of desirability level, $D$, remain constant over the time horizon $T$ for all members of a community. 
Under full knowledge conditions, an individual $j$ (or its complement $c$ ) is assumed to correctly know the measures $E$ and $D$ characterizing $i$. Under symmetry in knowledge conditions, individual $i$ fully knows the corresponding measures for $j$ and $c$. In case partial knowledge is built into the model, then $j$ (and $c$ ) might be thought of as possessing incorrect but approximate measures of $E$ and $D$ for $i$, at any point in time $t(n)$. Asymmetry would, in turn, imply that these approximations differ, and that they evolve independently from one another.

A further mechanical simplification is in order, at this point: to more effectively window into the interaction on hand between individuals $i$ and $j$, the background influences can be assumed to be negligent, so that the terms ${ }^{+} U_{i j j, c}[t(n)],-{ }^{-} U_{c, i l j}[t(n)]$ can be omitted from the kinematic conditions. These simplifying assumptions imply, among other things; that the exogenous shocks (noise, disturbance) imposed on the ( $i j)$ interaction could be either of the "hard" or "soft" variety, without one being able to necessarily distinguish among the two at this stage.

As a result of the above stated conditions, the following equations become operative:

$$
\begin{aligned}
& U_{i}[t(n)]=(\gamma-1)^{-} U_{i, j}\left[t(n)+^{+} U_{i, j}[t(n)]=H_{i}\right. \\
& U_{j}[t(n)]=(\gamma-1)^{-} U_{j, i}\left[t(n)+^{+} U_{j, i}[t(n)]=H_{j}\right.
\end{aligned}
$$

Upon substitutions, and through the further assumption that the terms within absolute value brackets have been replaced with squared ones, see Appendix, the following symmetric conditions are obtained for parties $i$ and $j$ in the exchange:

$$
\begin{gathered}
(\gamma-1)\left\{\alpha\left(s_{j, i}[t(n)]-E_{j, i}[t(n)]\right)^{2}+\beta\left(s_{j, i}[t(n)]\right.\right. \\
\left.\left.-D_{j, i}\right)^{2}\right\}-\delta\left(s_{i, j}[t(n)]-s_{i, j}[t(n-1)]\right)^{2}=H_{i} \\
(\gamma-1)\left\{\alpha\left(s_{i, j}[t(n)]-E_{i, j}[t(n)]\right)^{2}+\beta\left(s_{i, j}[t(n)]\right.\right. \\
\left.\left.-D_{i, j}\right)^{2}\right\}-\delta\left(s_{j, i}[t(n)]-s_{j, i}[t(n-1)]\right)^{2}=H_{j}
\end{gathered}
$$

which, in turn and upon notational simplifications (time designations have been eliminated and the $t(n-1)$ time period substituted simply with $(-1)$, and substitution of constants) produce:

$$
\begin{aligned}
& {\left[s_{j, i}-E_{i}\right]^{2}+c_{1}\left[s_{j, i}-D_{i}\right]^{2}-c_{2}\left[s_{i, j}-s_{i j}(-1)\right]^{2}} \\
& \quad=c_{3, i} \\
& {\left[s_{i, j}-E_{j}\right]^{2}+c_{1}\left[s_{i, j}-D_{j}\right]^{2}-c_{2}\left[s_{j, i}-s_{j, i}(-1)\right]^{2}} \\
& \quad=c_{3, j}
\end{aligned}
$$

with the new constants given by:

$$
\begin{aligned}
c_{1} & =\beta / \alpha, \quad c_{2}=\delta / \alpha(\gamma-1) \\
c_{3, i} & =H_{i} / \alpha(\gamma-1) \\
c_{3, j} & =H_{j} / \alpha(\gamma-1) .
\end{aligned}
$$

It is clear from conditions (17) and (18) that due to the specific formulation of the action reaction dynamics there is not time delay built into it, except for the expectations and last term in the left hand side of both conditions, terms which have to do with addictive behavior. More complicated formulations of an action-reaction system would have time delayed effects more abundantly found in conditions (17) and (18). This does not, however, in any way diminish the validity of the results to be demonstrated later.

Considering the relative magnitude of the "security" enclave $D_{1} D_{2}$ of Fig. $1 \mathrm{~b}$, one could present conditions for response strategy switching. But, again, consideration of these (upper and lower) limits in affecting the magnitude of the signal sent at $t(\mathrm{n}+1)$ is left to future research.

\subsubsection{Dynamic Equilibrium Points}

Dynamic equilibrium, at the individual level, is obtained when a constant in magnitude signal size is emitted in time, i.e. when addiction (habit, tradition) is complete and the disutility term due to variance in signal size drops out, as well as when expectations have reached a plateau in time:

$$
s_{i / j, j / i}[t(n+1)]=s_{i / j, j / i}[t(n)]=s_{i / j, j / i}^{*}
$$

The above conditions contain within them the following set of questions:

$$
\begin{aligned}
{\left[s_{j, i}^{*}-E_{i}\right]^{2}+c_{1}\left[s_{j, i}^{*}-D_{i}\right]^{2} } & =c_{3, i} \\
{\left[s_{i, j}^{*}-E_{j}\right]^{2}+c_{1}\left[s_{i, j}^{*}-D_{j}\right]^{2} } & =c_{3, j}
\end{aligned}
$$

with $D$ s denoting (initially set and fixed in time) desired targets, and $E$ s are (close to equilibrium) constants as a result of incessant learning. By abandoning the (*) notation, considering that $\left(1+c_{1}\right)$ is not zero, and by appropriate substitution of constants, see Appendix, one obtains a pair of second degree equations:

$$
\begin{aligned}
& s_{j, i}^{2}-k_{2, i} s_{j, i}+k_{3, i}=0 \\
& s_{i, j}^{2}-k_{2, j} s_{i, j}+k_{3, j}=0
\end{aligned}
$$

which supply a pair of equilibria for each party of an interaction $(i j)$, designated as $\left(S_{j, i}, 2 S_{j, i}\right.$ for entity $j$, and $\mathbf{S}_{i, j}$, ${ }_{2} S_{i, j}$ for entity $\left.i\right)$. For these two sets of equilibria to be real, 
the following must hold:

$$
\begin{aligned}
& k_{2, i}>4 k_{3, i}, \\
& k_{2, j}>4 k_{3, j},
\end{aligned}
$$

with a pair of identical equilibria emerging when conditions $(25 \mathrm{a}, \mathrm{b})$ operate as equalities.

It is immediately apparent that if constant $k_{3, i / j}$ is/are zero (not an impossibility by any means) then there is no interaction, at equilibrium, in the exchange $(i j)$. Since individuals $i$ and $j$ are not identical, the constants need not be equal, too, thus allowing for a rich variety of events to occur within an interaction. Moreover, and following on the realization that the constants in Eqs. $(25 \mathrm{a}, \mathrm{b})$ could be zero, one can derive conditions applicable to all possible exchanges within a community, such that all interaction among its members ceases, and as a result the community collapses.

Further, it is also immediately apparent that within this simple context, at dynamic equilibrium, the equilibrium signal size of player $i$ emitted towards $j$ is independent of the corresponding signal size from $j$ towards $i$. However, both sizes depend on each player's attributes as they are reflected in the values of the relevant parameters $\mathbf{k}$.

Eigenvalue analysis in the neighborhood of the equilibrium points in Eqs. (23) and (24) reveals their nature. i.e. whether they are stable or unstable, and the motion towards or away form them. As the motion towards equilibrium (or away from it in case of instability) is not necessarily nodal, there is constant over and under shooting of the equilibrium point in an oscillatory manner. Individual interaction is beset with cyclicality, and this event is a mere manifestation of the nonlinearity of human interchange.

At least one (and possibly both) dynamic equilibria (for both parties) is unstable, but the particulars of this analysis are to be presented in detail at another forum. Basically, the slope of the dynamic path close to the equilibrium (identifying the change in the current signal size given a unit change in the magnitude of the signal at the previous time period) must be in absolute value no greater than one. This condition is possible to be met at least at one point.

Standard nonlinear dynamical analysis suggests that the characteristic polynomial, evaluated close to the equilibrium neighborhood, picks the type of spiraling (cyclical) or nodal motion one encounters there. Such motion is closely tied to the specific values of the model's parameters, and both types are quite likely here. All conditions of individual interaction listed in the introductory section of the paper can find their qualitative replicas in the phase portrait and in parameter space of the above model.

Individual equilibrium conditions, when applied to all possible community interactions provide communitywide dynamic equilibrium. Most likely, this equilibrium is far from the origin in the state variable space, and thus the community is a viable one in time, for a very wide range of parameter values. Obviously, when all individual interactions have collapsed within a community, the community itself has collapsed. But, beyond this rare condition, one comes to wonder how viable could a community be when all interactions within it, through all individual exchanges, remain at a (non zero) steady state throughout dynamic equilibrium and in absence of exogenous shocks to refuel its evolution. This question raises the issue of how "reasonable" it is to identify "community equilibrium" as a state in which all individual exchanges are at equilibrium. This issue is left to future work to ponder.

One additional point of significance is this: although possible, chaotic dynamics are (even in theory, see Dendrinos (1992)), very unlikely in the above model. To show this, one ought to examine the likelihood that the period three cycles in the above stated dynamics has real solutions. Since the complicated forms involved in the iterative process (on both the $i$ and $j$ front) render the analysis almost intractable, one needs to resort to numerical simulations to locate (the rare) chaotic regimes. In qualitative terms, one detects that even this simple model formulation affords little chance that the roots of a three in degree equation are all real (and non negative, thus admissible).

\subsubsection{Analysis of Equilibria}

Of interest is the configuration of these two fixed points $\left({ }_{1} S_{i, j},{ }_{2} S_{i, j}\right)$ in the phase space, particularly when compared with the location of the (moving up till equilibrium) expectations, $E_{i}$, and the fixed desirability target, $D_{i}$, of an individual $i$ in the exchange $(i j)$.

Initially, the proximity to $D_{i}$ of the two fixed points is examined. Formulating the difference $\left({ }_{1} S_{i, j}-D_{i}\right)$ and looking at the parameter specifications that would render it close to zero, one detects (by suitable substitutions from Eqs. (A.11,2) and simple algebraic manipulations) that these fixed points are in general far from the target $D_{j}$. There are, however, rare levels of desire, which if coupled with a set of favorable parameter values do allow for the existence of satisfied dreamers. This occurs when:

$$
D_{i}^{2}-\lambda E_{i} D_{i}+\lambda\left[E_{i}-H_{i} / \alpha(\gamma-1)\right]=0
$$

where, $\lambda=4[1+\beta / \alpha]$. Dreamers' dual (because there is a pair of such roots) paradise exists if and when:

$$
D_{i}^{*}=(1 / 2)\left\{\lambda E_{i}^{*}+\left[\left(\lambda \mathrm{E}_{\mathrm{i}}^{*}\right)^{2}-4 \lambda \mu\right]^{1 / 2},\right.
$$

where, $\mu=E_{i}^{*}-H / \alpha(\gamma-1)$. However, not both of them are dynamically stable, and indeed it could be that none is.

What the above analysis implies is that first, cynics are much more likely to exist in a state of equilibrium than dreamers, although not necessarily without experiencing some surprises; and second, that dreamers are likely to be very unhappy in a (stable) community where vast ranges of desires remain unrealizable utopias. Of course, given 
enough time on the way towards equilibrium, the possibility exists in this evolutionary model that, by adjusting expectations, dreamers might be able to switch mode of behavior and convert to cynics thus avoiding deep disappointment.

\section{CONCLUSIONS}

In setting the building blocks of individual interaction dynamics, a set of basic elements were identified, a number of alternative edifices were constructed, and their stability properties analyzed. It was found that there are two types of individuals within a community of interacting agents, that of dreamers and cynics, with various kinds of expectations and desires governing their exchange with others. It is noted that in the field of psychology, there are numerous definitions (I have come across at least eight on each) of these two notions, namely "dreamer" and "cynic". The terms are used here in a manner distinct from any of these existing definitions (and they could be denoted, for example, as $D$-dreamer and $D$-cynic, for dreamer/cynic "according to Dendrinos")!

By choosing one among a set of alternative response strategies in reacting to signals received, it was established that individuals of either type follow dynamical paths of interfacing which could lead to either stable or unstable end states. In particular, under a tit-for-tat response strategy, the likelihood of unstable end states was revealed. It was realized that dreamers are very likely to end up being left with unrealized utopian states.

Although the model was presented as an individual interaction process with purpose and intent in the interchange, the structure is broad enough to accommodate any formulation associated with public policy actions. In this case, the model can be easily modified to account for the action of a public agent and the reaction of the socioeconomic system under governance.

As stated at the outset, the model formulation (being so generic, systemic and detailed in its exposition) lent itself to numerous extensions and modifications, all left to future work and the interested reader. Central among them is the introduction of time delays into the reaction process. Time delays are paramount, when of course space distances are considered in an exchange. Space also could be explicitly introduced by making signal size (sent or received) a function of distance (between sender and receiver). Lastly, transforming this Newtonian mechanics type actionreaction system to a relativistic or even quantum type interaction is certainly an interesting extension, too.

\section{Acknowledgements}

The Paper was Initially Written in October 1998. It is Dedicated to My Daughter Alexia Diana.

\section{References}

Bertuglia, C.S., Leonardi, G., Occelli, S., Rabino, G.A., Tadei, R. and Wilson, A.G. (1987) Urban Systems: Contemporary Approaches to Modelling (Croom Helm, London).

Bowlby, J. (1969) Attachment and Loss Attachment, (Basic Books, New York) 1.

Bowlby, J. (1973) Attachment and Loss Separation, (Basic Books, New York) 2.

Bowlby, J. (1980) Attachment and Loss Loss Sadness, and Depression, (Basic Books, New York) 3.

Dendrinos, D.S. (1992) "Bifurcations and Turbulence in Socio-Spatial Dynamics are Eccentric Events", Socio-Spatial Dynamics 3(2), $75-88$.

Dendrinos, D.S. (1998) "On the foundations of social dynamics: an efficient mathematical statement of a general framework underlying a complex nonlinear social determinism, incorporating a supraobserver and a suprastructure", In: Bertuglia, C.S., Bianchi, G. and Mela, A., eds, The City and its Sciences (Physica-Verlag, Heidelberg), pp 203-224.

Lucas, Jr, R.E. (1984) Studies in Business-Cycle Theory (MIT Press, Cambridge, Mass).

Rinaldi, S. and Gragnani, A. (1998) "Love Dynamics Between Secure Individuals: a modeling approach", Nonlinear Dynamics, Psychology, and Life Sciences 2(4), 283-302.

\section{APPENDIX}

The model conditions from Eqs. (13) and (14), by appropriate substitutions, are:

$$
\begin{gathered}
(\gamma-1)\left\{\alpha\left|s_{j, i}[t(n)]-E_{j, i}[t(n)]\right|+\beta \mid s_{j, i}[t(n)]\right. \\
\left.-D_{j, i} \mid\right\}-\delta\left|s_{i, j}[t(n)]-s_{i, j}[t(n-1)]\right|=H_{i} \\
(\gamma-1)\left\{\alpha\left|s_{i, j}[t(n)]-E_{i, j}[t(n)]\right|+\beta \mid s_{i, j}[t(n)]\right. \\
\left.-D_{i, j} \mid\right\}-\delta\left|s_{j, i}[t(n)]-s_{j, i}[t(n-1)]\right|=H_{j}
\end{gathered}
$$

with the substitution of the absolute values brackets with squared terms resulting in:

$$
\begin{aligned}
& (\gamma-1)\left\{\alpha\left(s_{j, i}[t(n)]-E_{j, i}[t(n)]\right)^{2}+\beta\left(s_{j, i}[t(n)]\right.\right. \\
& \left.\left.-D_{j, i}\right)^{2}\right\}-\delta\left(s_{i, j}[t(n)]-s_{i, j}[t(n-1)]\right)^{2}=H_{i} \\
& (\gamma-1)\left\{\alpha\left(s_{i, j}[t(n)]-E_{i, j}[t(n)]\right)^{2}+\beta\left(s_{i, j}[t(n)]\right.\right. \\
& \left.\left.-D_{i, j}\right)^{2}\right\}-\delta\left(s_{j, i}[t(n)]-s_{j, i}[t(n-1)]\right)^{2}=H_{j}
\end{aligned}
$$

which are the conditions (15) and (6a,b) of the main text. Notational simplifications produce:

$$
\begin{aligned}
& (\gamma-1)\left\{\alpha\left(s_{j, i}-E_{i}\right)^{2}+\beta\left(s_{j, i}-D_{i}\right)^{2}\right\} \\
& -\delta\left[s_{i, j}-s_{i, j}(-1)\right]^{2}=H_{i}
\end{aligned}
$$




$$
\begin{aligned}
& (\gamma-1)\left\{\alpha\left(s_{i, j}-E_{j}\right)^{2}+\beta\left(s_{i, j}-D_{j}\right)^{2}\right\} \\
& -\delta\left[s_{j, i}-s_{j, i}(-1)\right]^{2}=H_{j}
\end{aligned}
$$

where, the $t(n)$ notation has been dropped, the $t(n-1)$ notation has been substituted with $(-1)$, and the $E_{j, i}$ variable (the expectations $i$ has of $j$ 's signal directed towards $i$ ) has been simply been noted as $E_{i}$ (with similar modifications for $E_{i, j}, D_{j, i}$ and $\left.D_{i, j}\right)$. At a point of equilibrium, where conditions (21) and (2) hold, simple algebraic operations produce (as the $*$ has been dropped):

$$
\begin{aligned}
& \left(1+c_{1}\right) s_{j, i}^{2}-2\left(E_{i}+c_{1} D_{i}\right) s_{j, i}+E_{i}^{2}+c_{1} D_{i}^{2} \\
& -c_{3, i}=0 \\
& \left(1+c_{1}\right) s_{i, j}^{2}-2\left(E_{j}+c_{1} D_{j}\right) s_{i, j}+E_{j}^{2}+c_{1} D_{j}^{2} \\
& -c_{3, j}=0
\end{aligned}
$$

where expectations are fixed, as no further learning takes place. Further algebraic simplifications result in conditions (23) and (4) of the text, where the new set of constants are given by:

$$
\begin{aligned}
k_{2, i} & =2\left(E_{i}+c_{1} D_{i}\right) /\left(1+c_{1}\right), \\
k_{3, i} & =\left(E_{i}+c_{1} D_{i}-c_{3, i}\right) /\left(1+c_{1}\right) \\
k_{2, j} & =2\left(E_{j}+c_{1} D_{j}\right) /\left(1+c_{1}\right), \\
k_{3, j} & =\left(E_{j}^{2}+c_{1} D_{j}^{2}-c_{3, j}\right) /\left(1+c_{1}\right) .
\end{aligned}
$$

Each of two solutions, in the pair of second degree equations in conditions (23) and (4), acquires the expression:

$$
\begin{aligned}
& { }_{1} S_{j, i}=(1 / 2)\left\{k_{2, i}+\left[k_{2, i}^{2}-4 k_{3, i}\right]^{1 / 2}\right\}, \\
& { }_{2} S_{j, i}=(1 / 2)\left\{k_{2, i}-\left[k_{2, i}^{2}-4 k_{3, i}\right]^{1 / 2}\right\}
\end{aligned}
$$

and:

$$
\begin{aligned}
& { }_{1} S_{i, j}=(1 / 2)\left\{k_{2, i}+\left[k_{2, j}^{2}-4 k_{3, j}\right]^{1 / 2}\right\} \\
& { }_{2} S_{i, j}=(1 / 2)\left\{k_{2, j}-\left[k_{2, j}^{2}-4 k_{3, j}\right]^{1 / 2}\right\} .
\end{aligned}
$$




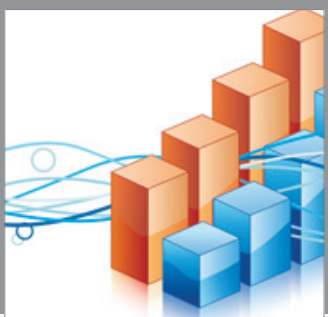

Advances in

Operations Research

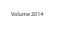

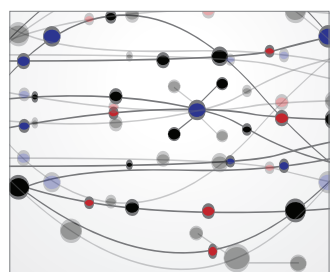

\section{The Scientific} World Journal
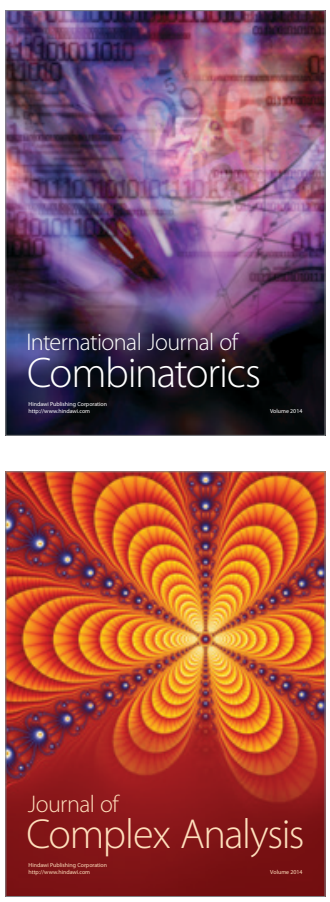

International Journal of

Mathematics and

Mathematical

Sciences
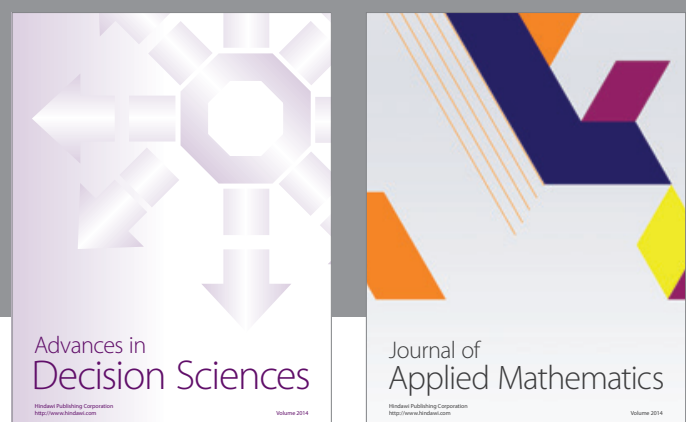

Journal of

Applied Mathematics
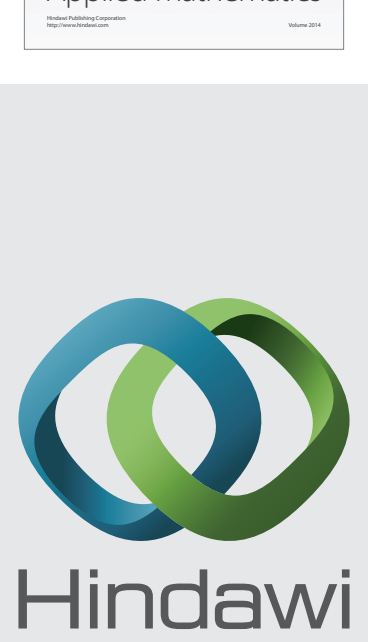

Submit your manuscripts at http://www.hindawi.com
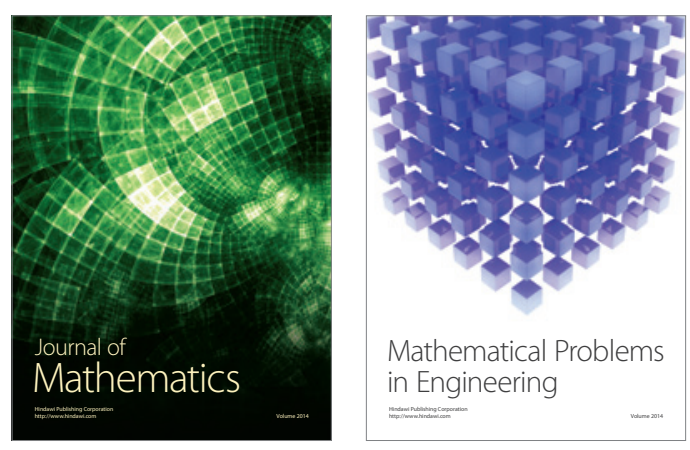

Mathematical Problems in Engineering
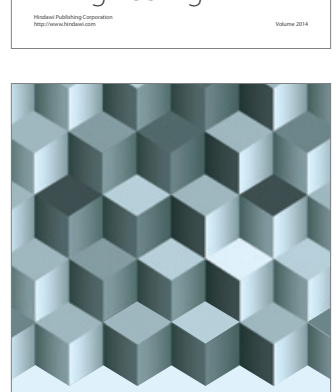

Journal of

Function Spaces
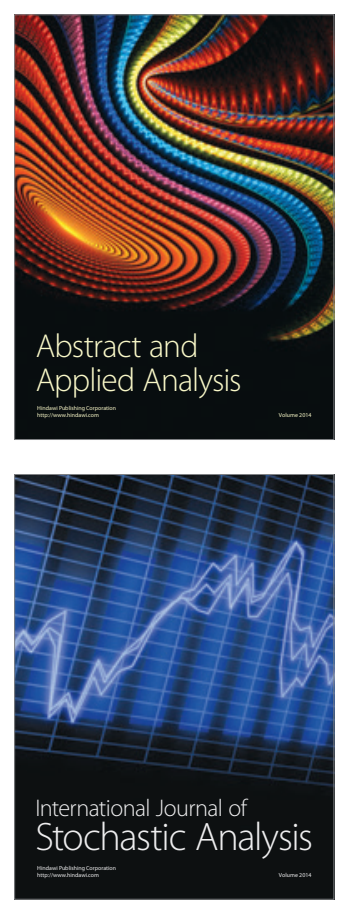

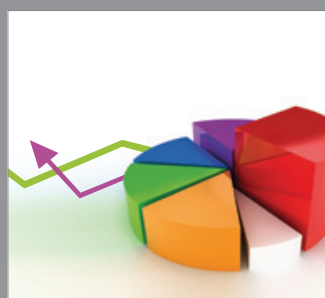

ournal of

Probability and Statistics

Promensencen
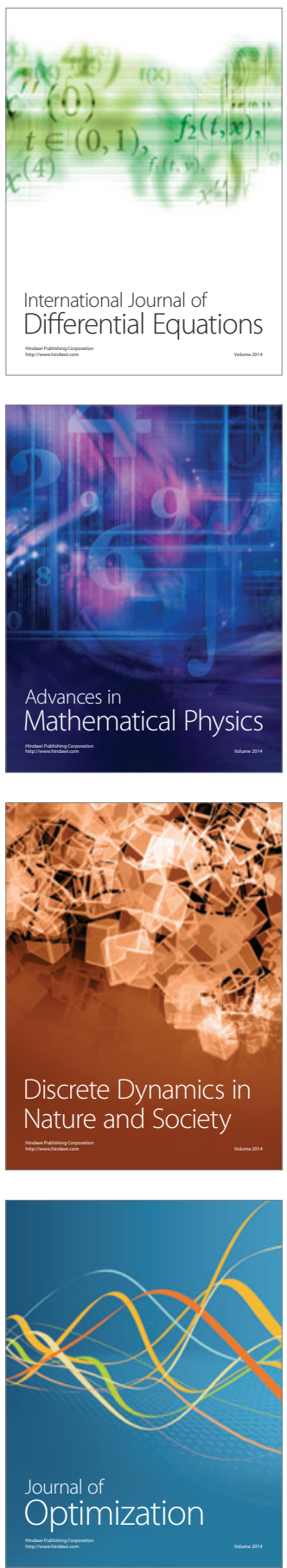